\title{
Generic Reusable Concern Compositions ${ }^{\star}$
}

\author{
Aram Hovsepyan, Stefan Van Baelen, Yolande Berbers, Wouter Joosen \\ Katholieke Universiteit Leuven, Departement Computerwetenschappen, Celestijnenlaan 200A, \\ B-3001 Leuven, Belgium \\ $\{$ Aram.Hovsepyan, Stefan.VanBaelen, Yolande.Berbers, \\ Wouter.Joosen\}@cs. kuleuven. be
}

\begin{abstract}
The increasing complexity of software applications requires improved software development techniques in order to cope with, a.o., software reuse and evolution, the management of heterogeneous features, and the retargeting of systems towards new SW platforms. The introduction of aspect-oriented software development (AOSD) and the support for model-driven development (MDD) are two important and promising evolutions that can contribute to a better controlling of software complexity. In this paper we present an AOM-based framework to promote and enhance the reuse of oblivious concern models. We focus on software design patterns, which represent complete solutions to recurring concernspecific problems. We have developed a prototype composition engine written in ATL that can be used to compose two concern models specified in UML.
\end{abstract}

\section{Introduction}

Aspect-Oriented Modeling (AOM) is a recent development paradigm aiming at modularizing and composing concerns within software models by using Model-Driven Development (MDD) techniques. In fact, it can be considered as a special case of an MDD approach. Following an AOM approach, we can model parts of a complete solution separately, and compose them using model composition mechanisms.

For AOM to bring an increase of efficiency in software development, we need to make sure that it is possible to write concern models once and reuse them in different contexts. If one has to model a concern and write a composition transformation every time from scratch, the gains of AOM will be diminished substantially. However, there is a need to define criteria that make a given concern reusable.

In this paper we define and characterize reusable concern models. We provide several key criteria that we believe are crucial in enhancing concern model reusability. We have developed a graphical framework that we can use to model structure and behavior of a certain type of concerns and specify their composition using a composition model. We have implemented a generic composition engine in ATL [1], which can compose UML concern models specified in our framework .

In section 2, we define the key requirements that are necessary to improve concern reusability. We also comment why current AOM approaches come short in achieving

\footnotetext{
* The described work is part of the EUREKA-ITEA MARTES project, and is partly funded by the Flemish government institution IWT (Institute for the Promotion of Innovation by Science and Technology in Flanders).
} 
some of these requirements. In section 3, we present the Generic Reusable Concern Compositions (GReCCo) approach in details. We then describe the different composition mechanisms of our approach. In section 4, we present a case study and apply our approach to it. In section 5, we evaluate how GReCCo improves reusability by tackling each of the requirements presented earlier. In section 6, we present some related work. In the last section, we conclude and outline future work.

\section{Concern Reuse}

A concern is an area of interest or focus in system. Concerns are the primary criteria for decomposing software into smaller, more manageable and comprehensible parts that have meaning to a software engineer.

There is no clear notion of reuse for concern models in MDD as opposed to other software engineering approaches. Hence, we need to define and characterize what makes a given concern model more or less reusable ${ }^{1}$.

\subsection{Requirements for reuse}

We define a reusable concern model as a known solution for a certain problem, which can be used in several contexts to produce required assets. Different contexts mean for instance different applications, projects, companies, domains, etc. However, using this definition we cannot measure how reusable a concern model is. That is why we introduce several more concrete qualities of a concern model and of an AOM approach as a whole, which we believe are most important in increasing reuse of concerns.

Maximum obliviousness: Concerns, represented by their models, should be defined independently from other concerns. The primary model, describes the functional core of a given application and should not be modeled with any other concern in mind, which might eventually be composed with it. Other concerns should also be modeled as modules that represent a complete solution on their own. The concern composition specification - all the relevant parameters, mappings, and any extra weaving directives - should be specified in a separate composition model.

Composition symmetry: We should be able to compose concerns not only with the main application (primary model), but also with each other, independently from the primary model. This will allow the reuse of concerns within other concerns.

Interdependency management: There are many (often hidden) interactions between the different concerns, since they are not always completely orthogonal to each other. Such concern interactions can be classified to one of the following five categories: dependency, mutual exclusiveness, alternatives, conflict and mutual influence [2]. Hence, it is essential to be able to declare a potential interdependency explicitly, and take it into account when composing different concerns. Otherwise a reused model may create an invalid composition by e.g., introducing a dependency that is never resolved or adding a conflicting set of concerns.

\footnotetext{
${ }^{1}$ By the term concern we refer to an area of interest or focus in a system. Concerns, typically captured in aspects, are the primary criteria for decomposing software into smaller, more manageable and comprehensible parts that have meaning to a software engineer.
} 
Note that concern interdependencies influence the obliviousness quality, i.e. interdependent concerns cannot be completely oblivious.

\subsection{Current approaches}

There are many AOM approaches, each pursuing a distinguished goal and providing different concepts as well as notations. We consider them categorized by the alignment to phases criteria introduced by Op de beeck et al. [3]:

AOM approaches aligned to implementation Some AOM approaches have no high level modeling concept that maps the design to the concerns identified during requirements phase. Typically they focus on modeling AOP concepts such as join points, pointcuts, advices, etc. [4,5,6,7]. Even though this allows the modeling of a given concern in a certain manner, these approaches remain too close to the implementation level. Such approaches typically use lightweight UML extensions, which is advantageous in terms of tools, since UML profiles are supported by almost any UML tool. However, these approaches do not score high given our reusability criteria. Even though the primary model can be oblivious from any other concerns that may modify it, the concerns themselves implicitly depend on the structure of the primary model. This means that obliviousness is only unidirectional. Almost all implementation centric approaches support composition asymmetry. Even though an asymmetrical composition allows us to model all concerns as separate entities, we can compose a given concern only with the primary model. It is impossible to compose concerns with each other. In addition, as far as we know, these approaches provide no means to declare and detect concern interdependencies.

AOM approaches independent from implementation Several other approaches $[8,9,10]$ are implementation independent and provide higher-level mechanisms for concern modeling and composition. These approaches by default score better concerning reusability as they allow a more abstract concern representation and composition. They are typically based on UML metamodel extensions, however, none offers appropriate tool support yet. Even though most of them implicitly support concern reuse to some extent, they do not explicitly focus on reuse. None of these approaches provides means to declare and detect concern interdependencies. Moreover, these AOM approaches use template mechanisms that constrain the concern (re)use. A template usually defines a set of formal parameters that should be instantiated with a set of actual parameters. Templates also make concerns implicitly dependent on a primary model as the latter must instantiate the template parameters with concrete elements.

In this paper we introduce a conceptual framework for representing concerns and specifying compositions with other concerns, which improves support for reuse by tackling each of the requirements listed in subsection 2.1. In addition, we reuse existing work [11] that solves the problem of declaring and detecting concern interdependencies. 


\section{Generic Reusable Concern Compositions (GReCCo)}

In this section, we first describe the general principle behind the GReCCo approach. Then we present the specifics of the composition of concern models. In addition, we discuss the problem of concern interactions and show how this can be tackled by integrating an existing solution into our approach.

\subsection{General Description}

The GReCCo approach is used to compose concern models. We represent each composition step as the Greek letter upsilon ( $\Upsilon$ ). The left and the right branches of the upsilon contain two concern models. In theory, we should make a separation between a primary concern (or base) and other concerns. The primary concern represents the (functional) core of the application. The rest of the concerns represent a solution to a specific problem that is modularized and at a latter stage composed with the primary concern. However, our approach is symmetric and in practice it is unimportant what kind of concern models we are dealing with.

In order to combine the concern models, we also provide a composition model that instructs the model transformation engine how the two models should be composed. Concern Models 1 and 2 (fig. 1) describe the structure and the behavior of the concerns using UML class and sequence diagrams respectively. The Composition Model, which also consists of UML class and sequence diagrams, specifies how the concern models are composed by defining all composition-specific parameters and their bindings. This assures a higher degree of obliviousness of the two concerns. Since the Composition Model defines the model composition, it is not independent as opposed to the source models, i.e., it is meaningful only if the related models exist as well.

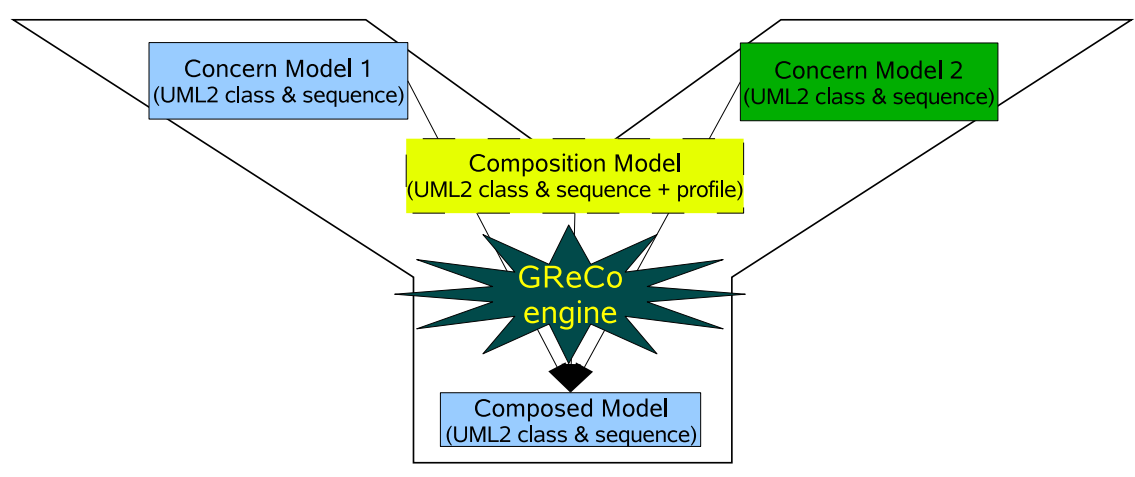

Fig. 1. General Approach

Elements that are not directly referenced in the Composition Model are copied to the Composed Model. Other elements are modified by the composition engine according to the specified composition strategy, which we will described in detail in the following 
section. Using a generic composition engine, we generate the output Composed Model from the composition and concern models.

Currently we have successfully applied our approach on design patterns by the Gang of Four (GoF) [12] and on security patterns [13].

\subsection{Composition Specification}

In order to compose two concern models, we need to specify the composition in the Composition Model. We consider the structural and behavioral concerns of the composition separately. Entities which are not involved in the composition specifications are copied to the Composed Model as they are, without any modifications. Examples of concern models and compositions specifics can be found in section 4 .

3.2.1 Structure From the point of view of one concern model, we distinguish three basic composition modifications that involve a single element: (1) we can add a new element, (2) we can modify the properties of an existing element, and (3) we can remove an existing element. As we are dealing with structural models, we discern between four main types of UML elements: class, property, operation and association. When two concern models are composed, there are some additional composition modifications that can occur to the input element(s). Two elements from different models can be merged to obtain a single entity with the combined properties. This merging is only relevant for class entities. Finally, some concerns introduce roles and/or template parameters as semantic variation points, which should be instantiated by using concrete UML elements. Hence, it is possible to indicate that a given model element is simply a template parameter, and must be instantiated by a concrete entity.

Add The composition of two concerns may need to add new elements. For instance, we might need to link two classes from the input concern models. In order to do so, we simply add the element to the mapping model and tag it with the UML stereotype $\ll a d d \gg$, which will indicate that this is a new element that must be added to the composed model.

Modify During the composition, we may need to modify some of the existing elements. For instance, we might need to rename an element because the same name is used in both input models. We specify this by marking the to be modified element with the $\ll$ modify $\gg$ stereotype. In addition, we indicate the UML meta-property, which needs to be modified, and its new value using a stereotype attribute (called a tag in UML 1.x). Each stereotype attribute will have a name, indicating the UML property that should be modified, a type, which is the same as the UML type of the property to be modified, and a value indicating the new value. For instance, if we wish to modify the name of a given class, we need to place a $\ll$ modify $\gg$ stereotype on the class, and fill out the name stereotype attribute with the new name of the class. If several UML properties of a given UML element need to be modified we will use several stereotype attributes on the same $\ll$ modify $\gg$ stereotype. 
Remove Due to the composition of concerns, certain entities may become unnecessary in the composed model. For instance, a concern may introduce an indirect association between two entities, which are already connected directly in another concern. We realize the removal of elements by putting the $\ll$ remove $\gg$ stereotype on the element that needs to be removed.

Merge When two concern models are involved, we sometimes have to deal with elements that represent a different view on the same entity. During the concern composition, we need to merge these elements to obtain a single entity with combined properties. In order to merge two elements, we need to add a UML association between the elements and mark it with the $\ll$ merge $\gg$ stereotype. The merge operation generates a new element that is composed from the properties of the original two elements. The name of the composed element is set to the concatenation of the names of the original elements. Conflicts such as name clashes, mutually exclusive properties, etc. should be resolved explicitly by using the modification strategy. Note that it is possible to merge more than two elements. In order to do this, we place binary $\ll$ merge $\gg$ associations between the classes.

Instantiate Often, a concern model represents a pattern that should be instantiated using elements from the other concern model. A pattern model usually contains a number of template elements (e.g., roles) that must be bound to concrete elements when the pattern is applied. In addition, a pattern model may contain a number of concrete elements that need to be introduced as new elements in the resulting model. As we aim for oblivious concerns, we model all pattern elements as concrete ones. On the composition model, however, we use the instantiation strategy, which tells the composition engine that a given concern element is a template that is instantiated by a concrete one. In practice, instantiation is similar to merge with the only difference that all conflicts are resolved by taking the properties of the concrete element. In addition, the name of the composed element is kept the same as the name of the concrete element. To specify an instantiation, we need to place an «instantiate $\gg$ dependency link from the entity that we consider as a template to the concrete one.

3.2.2 Behavior We believe that UML sequence diagrams are a very suitable representation for describing the behavior of a given concern, which has its structure specified by a UML class diagram. A concern model may contain several sequence diagrams. Each sequence diagram represents a certain scenario. Scenarios from the input concern models, which are completely independent of each other, are simply copied to the composed model. For behavior scenarios that should be composed, we need to be able to realize the following use-cases:

- specify the sequence of messages between the input behavior scenarios,

- indicate that a call from one input scenario is the same as a call from the other input scenario,

- replace a call or a set of calls from one of the input scenarios by a call or a set of calls from the other input scenario. 
In order to realize the first use-case, we introduce a notion similar to the general ordering relation from the UML 2.0 specification [14] (p. 466). A general ordering is a binary relation between two OccurrenceSpefications to describe that one of them must occur before the other one. Each OccurrenceSpecification contains a single event enclosed in an interaction fragment. We use a similar ordering concept that represents a directed binary relation between two sets of events, each enclosed in interaction fragments. The resulting scenario defines a partial ordering of the input events. To specify this on a model we use a dependency between the event(s) that should precede another event(s) and mark it with the $\ll$ genordering $\gg$ stereotype. The interpretation by the composition engine is that the dependency client fragment should immediately precede the dependency supplier fragment. Events that are not involved in any general ordering relation are put in parallel fragment blocks.

For the second use-case we use a dependency between the two calls marked with the $\ll$ merge $\gg$ stereotype. If we do not use this dependency the calls will be kept in duplicate. Note that event call merging is only possible when the call receiver classes are the same or are merged on the structural composition diagram.

Finally, to realize the last use-case, we group the event(s) that are to be replaced as well as the replacing events in interaction fragments. We place a $\ll$ replace $\gg$ dependency from the replacing to the to-be-replaced fragments.

Currently the UML 2.0 specification allows the grouping in interaction fragments only with an interaction operator chosen from a predefined set. In the first and last usecases we need a simple grouping without any interaction operator. To realize this we will use the loop interaction operator with a single iteration. Grouping in interaction fragments may be omitted in case it contains a single event or if the set of events is already grouped in another interaction fragment.

We illustrate some of these concepts on the example of two behavior concerns borrowed from [15]. Fig. 2 shows an example of the composition specification. The left part of the composition represents the behavior of an authentication concern. The right part describes the set of events for a logging concern. We would like to obtain a composed scenario that performs authorization and in case of a successful authentication writes it to a $\log$. If the authorization fails, the authorization concern itself is in charge of saving the failed attempt. The login() events from the two concerns are the same and we place a $\ll$ merge $\gg$ dependency link between them. In addition, we need to put the logEvent() event after the $O K$ message. We specify this by placing a $\ll$ genordering $\gg$ dependency from $O K$ to $\log E v e n t($ ). As a result we obtain a combined sequence scenario that performs both authentication and logging in case of a successful authentication. Note that in case we need to use the logging scenario after the authentication independent from the outcome we need to place the $\ll$ genordering $\gg$ dependency from the alt fragment to the $\log E v e n t()$ event.

\subsection{Concern Interactions}

Our approach can be used to obtain a system that is built by many concern compositions. Fig. 3 is an example of a composition tree that can be obtained if we combine more than one concern model. 


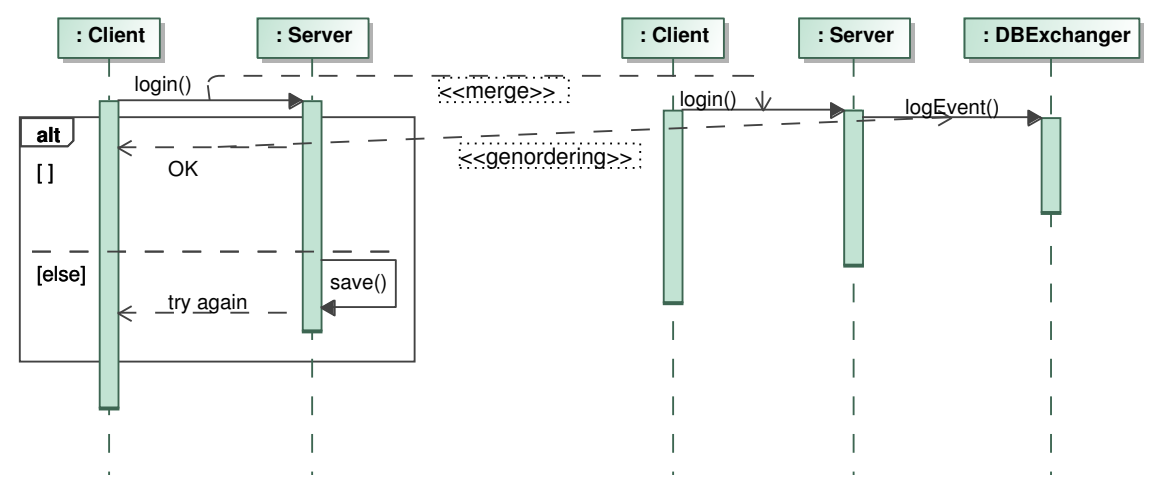

Fig. 2. Behavior Composition

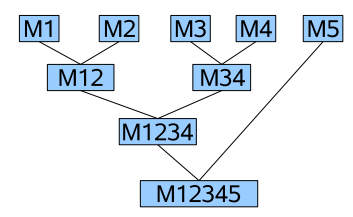

Fig. 3. Concern composition chain

However, concern models are rarely completely orthogonal to each other, but can relate to each other in a variety of different ways. A concern can be involved in an arbitrary number of interactions with one or more other concerns. Sanen et al. [11] distinguish between five different classes of concern interactions: dependency, conflict, choice, mutex and assistance. They also provide a conceptual framework for describing the relevant information about interactions between concerns that need to be captured. Their approach comes with a Concern Interaction Acquisition (CIA) prototype system that can be used to describe and query particular concern interactions. Fig. 4 shows an overview of the expert system. Domain experts add expertise about interactions between concerns to an OWL ontology using the Protégé [16] environment. The acquired knowledge is automatically transformed into a set of Prolog rules. The GReCCo tool has to provide a specification of a certain concern composition to be investigated for potential concern interactions. Based on this list, a set of Prolog facts is generated that contains all the predicate definitions that describe the listed concerns. Both the Prolog rules and Prolog facts are fed into a Prolog engine that through reasoning can detect all the interactions that occur in the given concern composition. This list of interactions is presented back to the GReCCo tool.

Our approach is currently constrained to the composition of only two concerns at a time. However, if we keep a composition history we could query about interactions with concerns that are already composed during the previous steps. This system is currently not incorporated in the GReCCo engine, however its integration is conceptually straightforward and will be realized in future versions. 


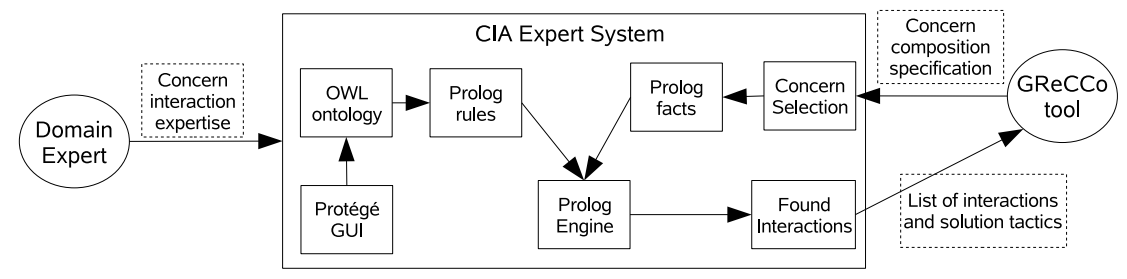

Fig. 4. Architecture of the CIA expert system

\section{Case-Study}

In this section, we present an application from the domain of Electronic Health Information and Privacy (EHIP). We start from a description of the primary model of the application. On top of this application, we apply several reusable concerns using the GReCCo methodology described in the previous section. Because of space restrictions some of the models will be shown only partially. For a complete description of this case study refer to [17].

\subsection{Screening Application}

Screening application represents an information system of a screening lab. Fig. 5 presents a UML class diagram for the screening lab application. Patients (ScreeningSubject) make an appointment to have their radiographic pictures (Screening) taken by a Radiographer. Two different Radiologists perform a Reading of the radiographic screening. In case the reading results are the same, an automatic Conclusion is generated. Otherwise, a third reading takes place, whereafter the third radiologist creates a final conclusion. In addition to the system itself, we have realized an additional client-server mechanism so that patients can consult their own data at home using, e.g., a web browser (Client). RegistryService offers a set of ScreeningServices, each having its own $i d$. Fig. 6 represents the scenario where patients execute a service in order to obtain their own data.

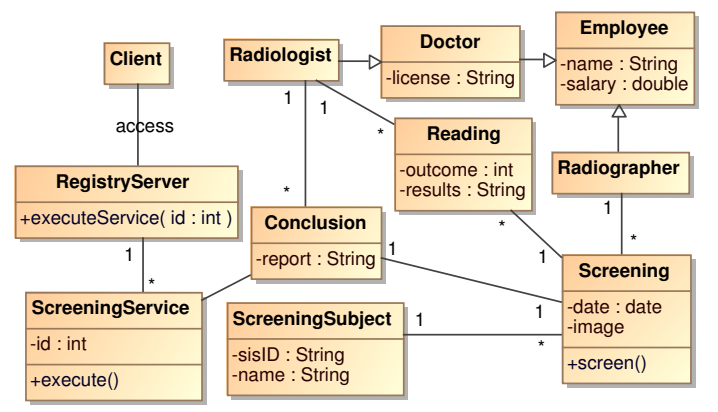

Fig. 5. Screening Lab Application Model

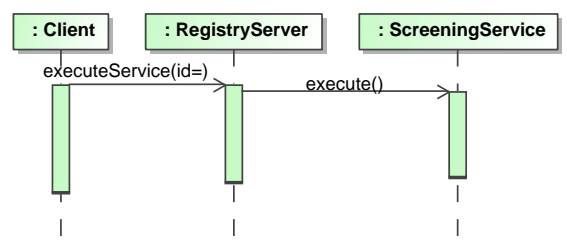

Fig. 6. Execute service 


\subsection{Application Firewall Pattern}

The registry server, introduced in the previous section, must ensure that only authenticated and authorized clients may use a given service. A sound solution in this case would be to interpose an application-level firewall that can analyze incoming requests for the registry services and check them for authorization. A client can access a service of the registry server only if a specific policy authorizes it. Policies for each application are centralized within the ApplicationFirewall and they are accessed through a policy authorization point (PAP) (fig. 7 and 8).

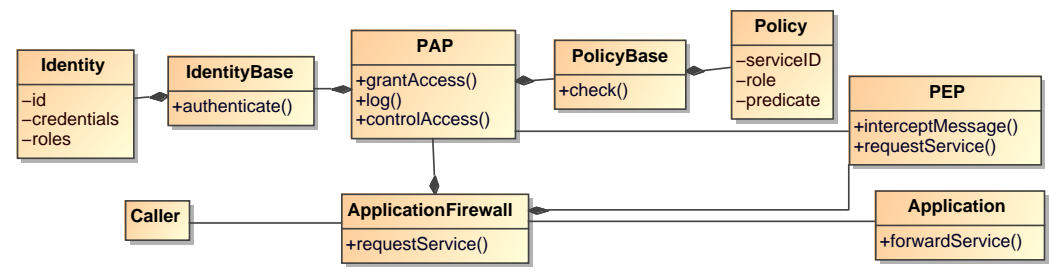

Fig. 7. Application Firewall Pattern

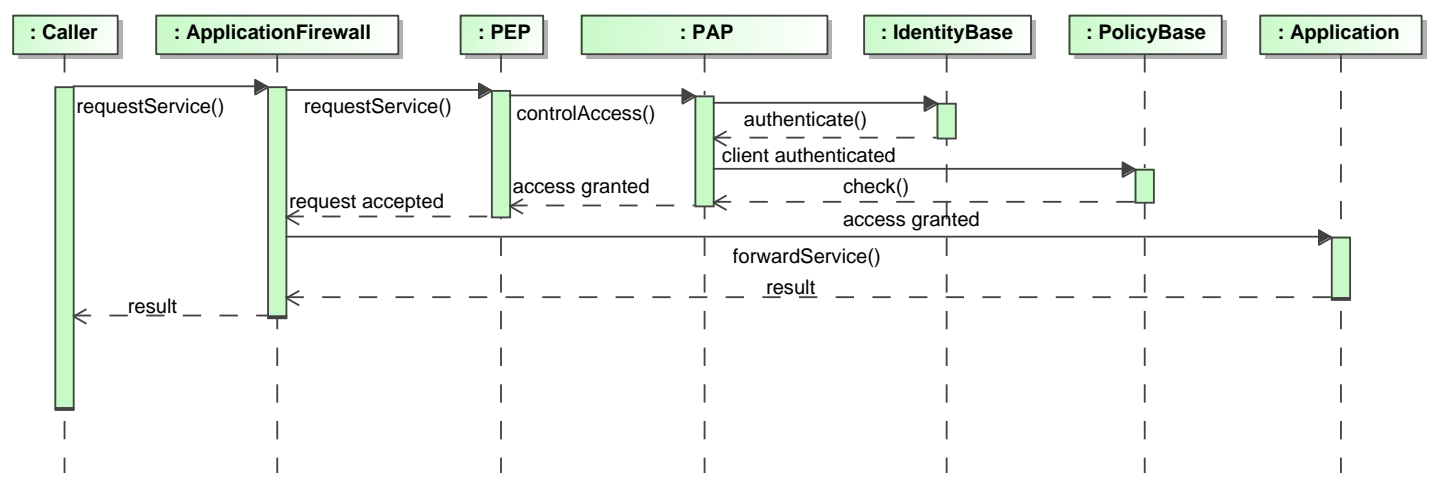

Fig. 8. Request Service

We want to compose the application firewall concern with the base. We follow the framework described in the previous section and define the composition model for the structure. We specify that Application, Caller and forwardService() should be instantiated by RegistryServer, Client and executeService() elements respectively. In addition, we need to remove the direct association between RegistryServer and Client as the application firewall concern will introduce an indirect link between the two. For illustration purposes, we rename the RegistryServer to Server by placing a $\ll$ modify $\gg$ 
stereotype on the class, using a tag name to indicate the new name (fig. 9). Because of space restrictions we do not show the composition specification of the behavior models. For that we would need to place a $\ll$ replace $\gg$ dependency link from a fragment, which includes executeService() and execute() events, to forwardService() event.

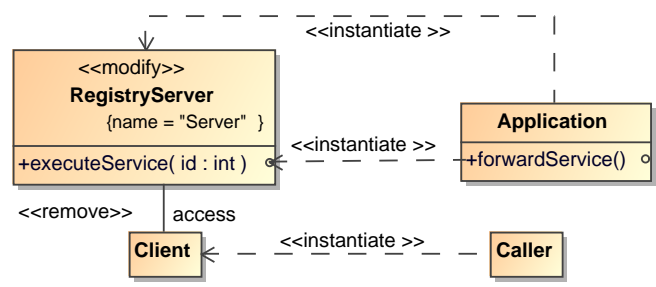

Fig. 9. Composition Model

The structure and behavior of the resulting composed model are partially shown on fig. 10 and 11 .

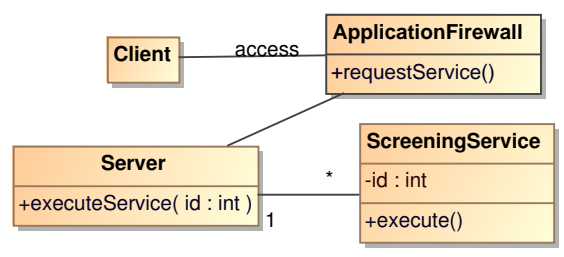

Fig. 10. Screening Application with Application Firewall Structure

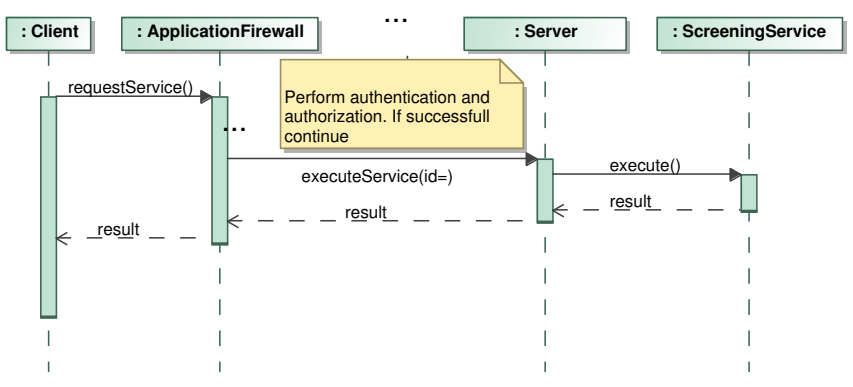

Fig. 11. Screening Application with Application Firewall Behavior

\subsection{Audit and Secure Logger Patterns}

In the next step of our case study we would like to add auditing support to our design. We have selected the Audit Interceptor pattern (fig. 12 and 13) to centralize auditing functionality. An Audit Interceptor intercepts business tier requests and responses and creates audit events.

Audit Interceptor depends on some secure logging facility without which it is impossible to guarantee the integrity of the audit trails. This is why we introduce also the Secure Logger pattern (fig. 14 and 15) that will ensure this additional requirement. 


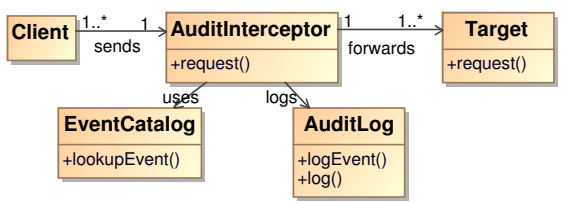

Fig. 12. Audit Interceptor Structure

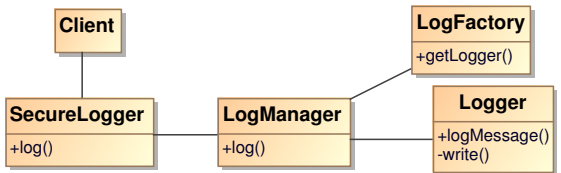

Fig. 14. Secure Logger Structure

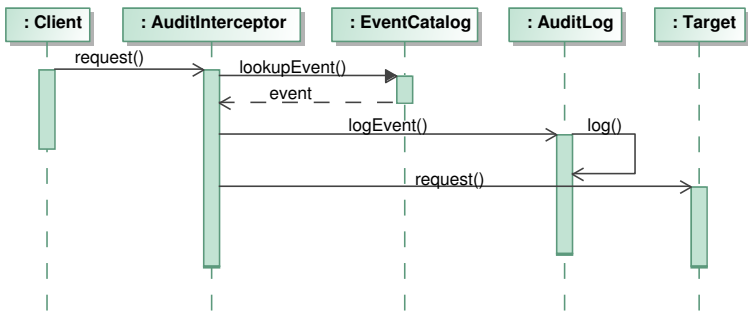

Fig. 13. Audit Interceptor Behavior

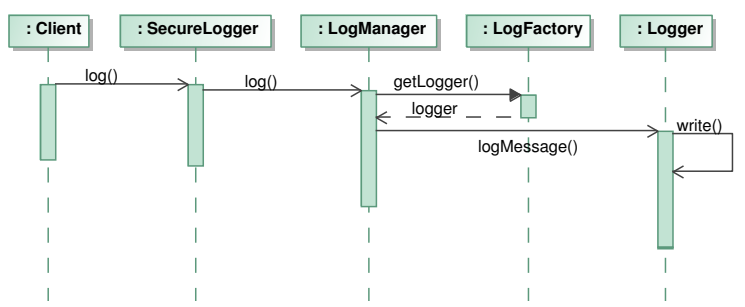

Fig. 15. Secure Logger Behavior

It is possible to apply these patterns to the application model (fig. 10) one by one. However, we chose to combine the two patterns using GReCCo, and apply the combined pattern on the application model. The AuditLog entity in the Audit Interceptor pattern represents the Client entity in the Secure Logger pattern. Hence, in order to combine the two patterns, we have to merge the two entities by relating them with a $\ll$ merge $\gg$ association (fig. 16). For the behavior composition we place a general ordering relation between the $\log ()$ event from the AuditLog object and the $\log ()$ event from the SecureLogger. The structure of the combined pattern is shown on fig. 17.

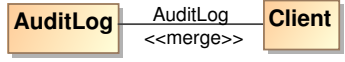

Fig. 16. Structure Composition Model

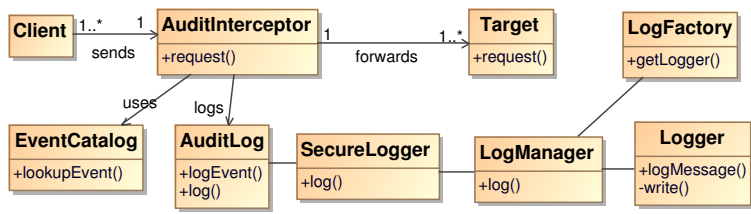

Fig. 17. Combined Structural Model 


\subsection{Final Refined Application}

In the final step we will compose the combined Audit and Secure Logging concerns (fig. 17) with the combined base and application firewall pattern (fig. 10). In order to realize the structural composition, we specify that the Client class from the combined pattern should be instantiated by the ApplicationFirewall class from the main application. We also instantiate the Target class by the Server class from the main application (fig. 18). Because of space restrictions we will not discuss the behavioral composition.

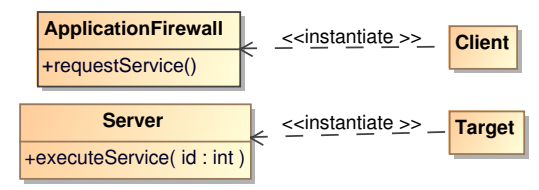

Fig. 18. Structure Composition Model

Fig. 19 shows the structure of the final application model with Application Firewall, Audit Interceptor and Secure Logger concerns composed into it.

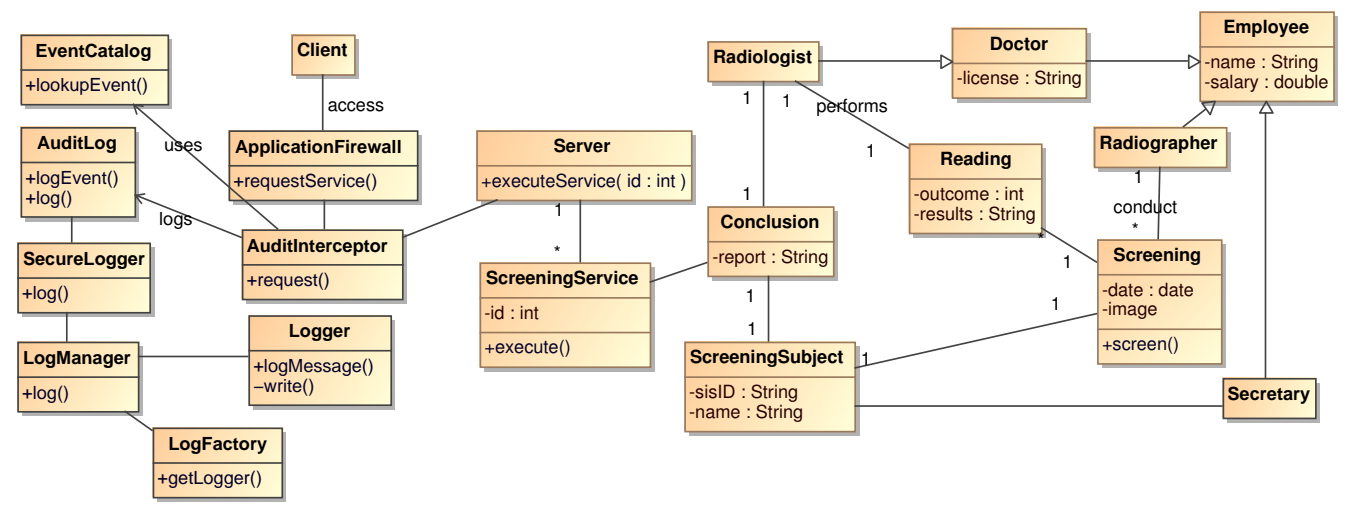

Fig. 19. Final Application Model

\section{Evaluation}

Our approach represents a framework for AOM using reusable concerns, whereby each concern is modeled using UML class and sequence diagrams. The approach comes with a prototype generic composition engine written in ATL, that can compose two given 
concern models. In this section, we evaluate how our approach can tackle the problems presented in section 2 .

\subsection{Proof-of-concept implementation}

We have developed a generic composition engine that realizes our approach on top of ATL. A current working version of GReCCo can be found in [18]. All the proposed mapping strategies for the structural composition have been implemented and evaluated with a number of examples. The behavioral composition is not yet fully supported.

\subsection{Key requirements for reuse}

We have illustrated on our case study how can we use the GReCCo approach to reuse patterns that modularize a given concern. Each of the concerns used in our case-study can be reused in another application simply by creating an appropriate composition model.

Maximum Obliviousness In our approach, each concern is modeled individually without any composition in mind. All the composition specifics, such as template parameters, structural and behavioral modifications, etc. are specified in a separate model, which is unique per composition. Given a certain concern, it is unaware of any other concerns that may modify its structure/behavior until the composition stage. We thus achieve a higher degree of obliviousness than the existing approaches.

Composition symmetry Our framework supports a generic approach to concern composition - i.e., symmetrical composition. We consider all the concerns of a system as equally important. Concerns can be composed not only with the main application, but also with each other.

Interdependency management A real-life system usually consists of many concerns, each tackling a specific part of the system. These concerns are rarely completely orthogonal, but rather influence each other in one or another way. Hence, concern interdependency management is a crucial point when reusing many concerns. We have tackled this requirement by reusing an existing generic framework that helps us detect and manage the different interactions when composing different concerns.

\section{Related Research}

The approach of Jacobson et al. [8] represents a use case driven software development method. AOSD with use cases comes with a systematic process that focuses on the separation of concerns throughout the software development life cycle. The approach provides different means of modularization that allows one to define application-generic modules. However, Jacobson's approach does not focus on reuse, nor does it provide any guidelines on how to reuse artefacts throughout different applications and projects. Moreover, there is no tool support available. 
The Theme approach of Clarke et al. [10] provides means for AOM in the analysis phase with Theme/Doc and in the design phase with Theme/UML. A Theme represents a modularized view of a concern in the system. It allows a developer to model features and aspects of a system, and specify how they should be combined.

The Aspect-Oriented Architecture Models (AAM) approach of France et al. [9] presents an approach for composing aspect-oriented design class models, where each aspect model describes a feature that crosscuts elements in the primary model. Aspect and primary models are composed to obtain an integrated design view. This approach is asymmetric and allows aspect models to be composed only with the primary model.

These two approaches are generic and support reuse to some extent. However, both approaches use a template-based mechanism to compose cross-cutting concerns. Each concern comes with a pre-defined set of template parameters and expects the other concern to have a certain structure or a certain functionality in order to match these. None of these approaches allows the definition and detection of concern interdependencies. Theme/UML is a symmetrical approach as any two concerns (or themes) can be composed. AAM is asymmetric, which further limits its reusability.

Klein et al. [15,19] present an approach for specifying reusable aspect models that define structure and behavior. The approach allows expressing aspect dependencies and weaving them in a dependency-consistent manner. However, it is possible to specify and detect only one sort of relationship between aspects, namely dependency. Klein's approach is symmetric and aspects can be composed with each other. This approach uses a template-based mechanism similar to the previous two approaches for the structural composition. The behavioral composition is specified using a semantic composition algorithm. Join points are specified using a pointcut, which can be seen as a small pattern scenario. Every match of this pattern against the main scenario is a join point. Speaking in the terminology of this approach, we use an explicit join point selection. We believe that in most concern composition scenarios the pointcut will match against a very few and possibly one join point. Moreover, Klein et. al. restrict the context where the concern can be used by defining a pointcut, which pertains to the concern. In our approach the join point can vary and is defined during the composition.

\section{Conclusions and Future Work}

In this paper we have listed and discussed what we believe are key characteristics for the enhancement of concern model reuse: obliviousness, composition symmetry, concern interdependency management. We have described a new approach for specifying concerns and their compositions and illustrated on a case-study from the EHIP domain. We have evaluated how the GReCCo approach can help us tackle each of the key qualities for improving reuse.

In the future we plan to build a library of concerns and evaluate the approach on a greater scale. We will improve the GReCCo engine to fully support the behavioral composition. Moreover, we are planning to add traceability support to our approach. 


\section{References}

1. F. Jouault, I. Kurtev: Transforming models with ATL. In: Proceedings of the Model Transformations in Practice Workshop at MoDELS, Montego Bay, Jamaica (2005)

2. F. Sanen, E. Truyen, W. Joosen, A. Jackson, A. Nedos, S. Clarke, N. Loughran, A. Rashid: Classifying and documenting aspect interactions. In: Fifth AOSD Workshop on ACP4IS. (2006)

3. S. Op de beeck, E. Truyen, N. Boucké, F. Sanen, M. Bynens, W. Joosen: A study of aspectoriented design approaches. In: Technical Report CW435, Department of Computer Science, Katholieke Universiteit Leuven. (2006)

4. D. Stein, S. Hannenberg, R. Unland: Designing aspect-oriented crosscutting in UML. In: Proc. of the 1st Workshop on AOM with UML. (2002)

5. R. Pawlak, L. Seinturier, L. Duchien, L. Martelli, F. Legond-Aubry, G. Florin: Aspectoriented software development with java aspect components. In: Aspect-Oriented Software Development. (2005) 343-369

6. L. Fuentes, P. Sánchez: Execution of aspect oriented uml models. In: ECMDA-FA. (2007)

7. T. Cottenier, A. van den Berg, T. Elrad: The motorola WEAVR: Model weaving in a large industrial context. In: Proc. of the 6th Int. Conf. on AOSD, Vancouver, Canada. (2007)

8. I. Jacobson, P-W. Ng: Aspect-Oriented Software Development with Use Cases. AddisonWesley Professional (2004)

9. Y. R. Reddy, S. Ghosh, R. B. France, G. Straw, J.M. Bieman, N. McEachen, E. Song, G. Georg: Directives for composing aspect-oriented design class models. In: LNCS 3880, p 75-105, Springer-Verlag. (2006)

10. E. Baniassad, S. Clarke: Aspect-Oriented Analysis and Design: The Theme Approach. Addison-Wesley (2005)

11. F. Sanen, E. Truyen, W. Joosen: Managing concern interactions in middleware. In: Distributed Applications and Interoperable Systems. (2007) 267-283

12. E. Gamma, R. Helm, R. Johnson, J. Vlissides: Design Patterns: Elements of Reusable ObjectOriented Software. Addison-Wesley Professional; 1st edition (1995)

13. K. Yskout, T. Heyman, R. Scandariato, W. Joosen: (A system of security patterns (technical report 04/12/06))

14. OMG: UML superstructure, v2.0. OMG Document number formal/05-07-04 (2005)

15. J. Klein, F. Fleureyand, J.-M. Jézéquel: Weaving multiple aspects in sequence diagrams. In: Transactions on Aspect-Oriented Software Development III. (2007)

16. The Protégé Ontology Editor and Knowledge Acquisition System: (http://protege.stanford.edu)

17. A. Hovsepyan, S. Van Baelen, Y. Berbers, and W. Joosen: Generic reusable concern compositions (GReCCo): Description and case study. Technical Report CW 508, Department of Computer Science, K.U.Leuven (2008)

18. Engine, G.R.C.C.: (http://www.cs.kuleuven.be/ aram/implementation.html)

19. J. Klein, J. Kienzle: Reusable aspect models. In: Proc. of the 11th Int. Workshop on AOM. (2007) 